\title{
Bridging the Gap - Connecting Single-Use Sensors to Standard Controllers
}

S. C. Kaiser ${ }^{1}$, F. Fietz ${ }^{1}$, N. Steiger ${ }^{1}$, D. Eibl ${ }^{1}$, M. Büring 2 , S. Beutel2 ${ }^{2}$ T. Scheper ${ }^{2}$, F. Beutler ${ }^{3}$, G. John ${ }^{3}$ 1) Institute of Biotechnology, Department of Liffe Science and Facility Management, Zurich University of Applied Sciences, Switzerland; 2) Institute of Technical Chemistry, Leibniz University Hanover, Germany; 3) PreSens Precision Sensing GmbH, Germany

\section{Introduction}

Since the first practical applications in the eighties, optrodes have gained increased interest in industry and academia. Monitoring important culture parameters is indispensable for bioprocess development and production. Therefore, single-use systems require integrated solutions. Optrodes are perfectly suited as they can be mounted into the plastic material. In addition, they can be pre-calibrated. Therefore we developed a new transmitter.

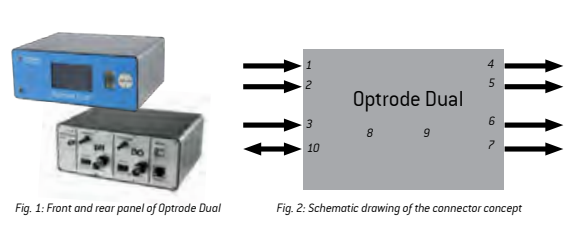

The basic connector concept of this prototype is shown in figure 2. It contains 2 SMA (1 and 2) connectors for fiber optics of the $\mathrm{pH}$ and $\mathrm{DO}$ sensor, electrical outlet 3 for the temperature sensor, 2 connectors for the electrochemical signals(ECS) (DO: 4 and $\mathrm{pH}: 5$ ], 2 connectors for $4-20 \mathrm{~mA}$ output ( $\mathrm{DO}: 6$ and $\mathrm{pH}: ?$ ) and an integrated barcode reader 8 for easy access of measurement settings. The LCD display 9 will inform about system status. In addition, a USB port 10 is planned for service functionality.

\section{Validation Study}

Basic characterization was done with phosphate buffered saline (PBS) as model medium.

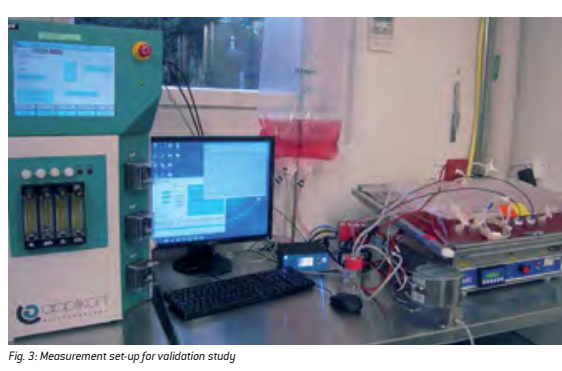

The Optrode Dual was connected to CultiBag RM 20 L optical (Sartorius Stedim Biotech) via polymer optical fiber. Data for $\mathrm{pH}$ and dissolved oxygen readings were transferred to the ezcontrol $^{\oplus}$ (Applikon, Netherlands, Fig. 3). Online measured pH values were compared to offline measurements as well. The Optrode Dual prototype was connected to the ez-control ${ }^{\circledR}$. Data collection of $\mathrm{pH}$ and $\mathrm{DO}$ readings was realized with the BioXpert software (version 2.93.122b2). The cultivation bag was placed on a BioWave 20 SPS platform (Wave Biotech, Switzerland).
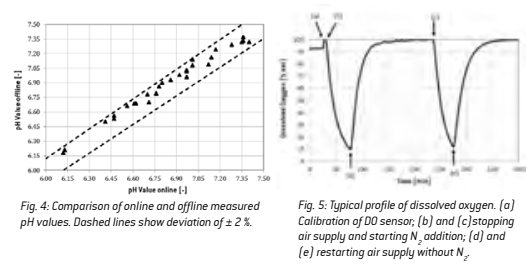

In total 5 repetitions of $\mathrm{CO}_{2}$ gasing were performed. The relative deviation between online and offline measured $\mathrm{pH}$ values was below $2 \%$ so both methods showed very good accordance (see Fig. 4). Figure 5 shows a typical DO profile when introducing $\mathrm{N}_{2}$ and air periodically in the bioreactor. Again 5 repetitions were made. Reference measurements with another system were not performed during this test.

\section{Microbial Cultivation}

E. coli K12 cultivations in LB-Medium were performed in a stainless steel bioreactor.

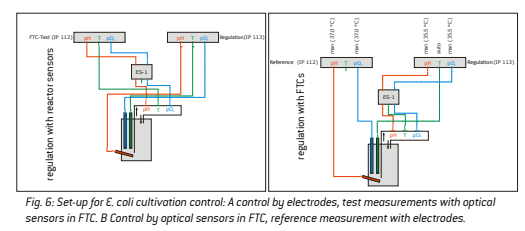

Reference $\mathrm{pH}$ and DO probes were mounted in the bioreacto while the optical sensors integrated in flow-through cells (FTCs] were applied in a bypass. pH and oxygen in the cultivation were controlled using the sensor probes installed in the bioreactor. Than the optical sensors were used for oxygen and $\mathrm{pH}$ control in the bioreactor.

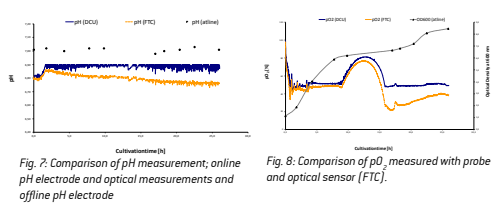

Fig 8 shows the resulting DO measurement while cultivation control was realized with measurement taken with probes inside the bioreactor. Some discrepancy was found. A potential reason could be lower oxygen in the bypass. Figure 7 displays $\mathrm{pH}$ measurements in the cultivation controlled by the optical pH measurement. In the following test cultivation control was conducted using $\mathrm{pH}$ and oxygen readings of the optical sensors installed in the bypass. Parameterization was changed during the first hours of cultivation in order to compensate for the maximum sampling rate of 30 s. Again differences in measurement values could be caused by the set-up and the optical sensors being positioned in a bypass.

\section{Cell Cultivation}

The Optrode Dual was evaluated for its functionality for a cultivation of suspension $\mathrm{CHO}$ cells.

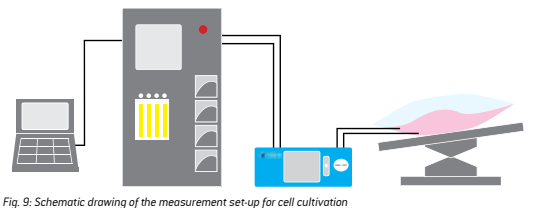

The set-up was similar to the validation study. The cultivation bag was filled with $1.5 \mathrm{~L}$ cell culture medium (CHO Master HP1, Cell Culture Technologies) 3 hours prior to inoculation, and tetracycline $\left[2.5 \mathrm{mg} / \mathrm{L} \mathrm{L}^{-1}\right]$ and Pluronic $(2 \mathrm{mg} / \mathrm{l})$ were added to the medium. The medium was conditioned to $37^{\circ} \mathrm{C}$ and aerated till it was saturated. Cell count and determination of cell viability were performed automatically with NucleoCounter NC-100 (chemometec, Denmark). Furthermore, $\mathrm{pH}$ was measured offline with a pH meter (Mettler Toledo, Switzerland).

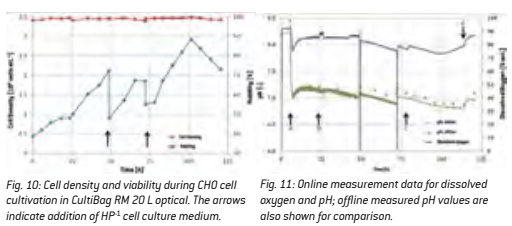

Online recorded data for dissolved oxygen (DO) and pH are shown in Fig. 11. Several instances were detected by the system: a] Recalibration was done after 6 hours to correct an operator error and $\mathrm{CO}_{2}$ gassing was corrected. b) A further increase in the DO level was recorded after 24 hours when gassing and movement of the bioreactor were changed. c) After $7 ?$ hours of cultivation of the bioreactor were changed. c) After ?
another increase in DO could be investigated, caused by the second increase in $\mathrm{CO}_{2}$ gassing to $0.5 \mathrm{~S} / \mathrm{pm}$. d) The last increase in DOvv was recorded after 110 hours of cultivation time, when $\mathrm{CO}_{2}$ supply was turned off. During the whole cultivation acceptable differences of below $0.2 \mathrm{pH}$ units could be detected between online and offline measured values, with offline determined values always being higher. This was probably caused by the time difference between sampling and measurement, during which $\mathrm{pH}$ regulating $\mathrm{CO}_{2}$ might have escaped the medium and caused $\mathrm{pH}$ to increase.

\section{Overall Conclusion}

The prototype transmitter Optrode Dual was successfully evaluated for basic functionality, microbial and cell cultivation. Two different cultivation vessels - stainless steel fermenter and disposable bioreactor - were tested. In both reactors the data was transferred correctly. Modifications of the controller settings became necessary with the microbial cultivation. Due to the different sampling rates.

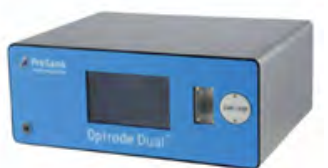

